\title{
Percepção das mães na utilização de métodos não farmacológicos para alívio da dor
}

\section{em lactentes}

\author{
Mothers' perception of the use of non-pharmacological methods for pain relief in nurslings \\ Percepción de las madres em el uso de métodos no farmacológicos para el alivio del dolor en
}

lactantes

Recebido: 22/05/2021 | Revisado: 29/05/2021 | Aceito: 02/06/2021 | Publicado: 18/06/2021

\author{
Carolina Cardoso Pires \\ ORCID: https://orcid.org/0000-0002-9580-5340 \\ Universidade Federal de Santa Catarina, Brasil \\ E-mail: carol_c_pires@hotmail.com \\ Patricia Klock \\ ORCID: https://orcid.org/0000-0002-2055-9720 \\ Universidade Federal de Santa Catarina, Brasil \\ E-mail: patricia.klock@ufsc.br \\ Roberta Costa \\ ORCID: https://orcid.org/0000-0001-6816-2047 \\ Universidade Federal de Santa Catarina, Brasil \\ E-mail: roberta.costa@ufsc.br \\ Ariane Thaise Frello Roque \\ ORCID: https://orcid.org/0000-0001-8637-0325 \\ Universidade Federal de Santa Catarina, Brasil \\ E-mail: ariane.frello.roque@ufsc.br \\ Bianca Bertotti Sonaglio \\ ORCID: https://orcid.org/0000-0002-5806-4299 \\ Universidade Federal de Santa Catarina, Brasil \\ E-mail: biancabs.enfermagem@gmail.com \\ Mariana Medeiros Sell dos Santos \\ ORCID: https://orcid.org/0000-0003-2916-0449 \\ Universidade Federal de Santa Catarina, Brasil \\ E-mail: marianacm31.1996@gmail.com
}

\begin{abstract}
Resumo
O objetivo do estudo foi compreender quais as percepções atribuídas pelos pais em relação ao manejo da dor durante a vacinação de seu filho, em uma clínica privada de vacinação do município de Florianópolis. Trata-se de uma pesquisa com abordagem qualitativa, através da Teoria Fundamentada em Dados. Os dados foram coletados com 10 mães no período de junho a agosto de 2019, por meio de entrevistas semi-estruturadas, gravadas e individuais em uma clínica de imunização privada localizada no município de Florianópolis/SC. Emergiram como resultados três categorias que fomentam o fenômeno central da pesquisa "Percepção das mães ao utilizar a amamentação como método não farmacológico para alívio da dor em crianças submetidas à vacinação". Através desse estudo, podemos identificar que os métodos não farmacológicos para alívio da dor, por mais que repercutem em satisfação e diminuição da angústia materna durante este momento, ainda são pouco utilizados na prática clínica. Conclui-se que tais métodos ainda são poucos estudados, apesar de possuírem grande valia para o alívio da dor e de traumas para os lactentes e seus respectivos pais e/ou cuidadores. Devendo-se valorizar a relevância do enfermeiro no contexto da vacinação, uma vez que, seus conhecimentos práticos-teóricos são peças fundamentais para que se obtenha o sucesso no manejo da dor.

Palavras-chave: Recém-nascido; Enfermagem; Vacinas; Aleitamento materno; Dor.
\end{abstract}

\begin{abstract}
The objective of the study was to comprehend the perceptions parents possess in relation to pain management during their child's vaccination, in a private vaccination clinic in the city of Florianópolis. This is a research with a qualitative approach, through Grounded Theory. Data were collected from 10 mothers from June to August 2019, through semi-structured, recorded and individual interviews in a private immunization clinic located in the city of Florianópolis / SC. Three categories emerged as results that foster the central phenomenon of the research "Mothers' perception when using breastfeeding as a non-pharmacological method for pain relief in children undergoing vaccination". Through this study, we can identify those non-pharmacological methods for pain relief, as much as they reverberate in the increase of satisfaction and in the decrease of maternal distress during this moment, are still underused in clinical practice. It concludes that such methods still are rarely studied, despite having great value for the relief of pain and trauma for nurslings and their respective parents and/or caregivers. The nurse's importance, in the
\end{abstract}


context of vaccination, should be valued since its practical theoretical knowledge is fundamental pieces for the success in the management of pain.

Keywords: Newborn; Nursing; Vaccines; Breastfeeding; Pain.

\section{Resumen}

El objetivo del estudio fue conocer las percepciones atribuidas por los padres en relación al manejo del dolor durante la vacunación de sus hijos, en una clínica de vacunación privada en la ciudad de Florianópolis. Es una investigación con enfoque cualitativo, a través de "Grounded Theory". Se recolectaron datos de 10 madres de junio a agosto de 2019, a través de entrevistas semiestructuradas, grabadas e individuales en una clínica de inmunización privada ubicada en la ciudad de Florianópolis/SC. Surgieron como resultados tres categorías que fomentan el fenómeno central de la investigación "Percepción de las madres al utilizar la lactancia materna como método no farmacológico para el alivio del dolor en niños vacunados". A través de este estudio, podemos identificar que los métodos no farmacológicos para el alivio del dolor, por mucho que afecten la satisfacción y disminuyan el malestar materno en este momento, aún son poco utilizados en la práctica clínica. Se concluye que estos métodos aún están poco estudiados, a pesar de tener un gran valor para el alivio del dolor y trauma de los bebés y sus respectivos padres y / o cuidadores. Debe valorar la importancia del enfermero en el contexto de la vacunación, ya que sus conocimientos prácticos-teóricos son fundamentales para lograr el éxito en el manejo del dolor.

Palabras clave: Recién nacido; Enfermería; Vacunas; Amamantamiento; Dolor.

\section{Introdução}

Uma das medidas de saúde pública mais importantes na prevenção de doenças é a vacinação. A primeira campanha de vacinação em massa realizada no Brasil foi em meados de 1904, idealizado por Oswaldo Cruz, o fundador de saúde pública no país tinha como principal objetivo controlar a varíola, doença esta que dizimava grande parte da população do estado do Rio de Janeiro (Brasil, 2003).

Atualmente, a varíola é a única doença já erradicada mundialmente, sendo seu último registro de incidência em 1977. Ainda assim, o sanitarista Oswaldo Cruz, deixou modelos de ações que hoje inspiram o Programa Nacional de Imunização (PNI). A vacinação é vista como uma das maiores estratégias na saúde pública do Brasil, visto que, em um país com média de 180 milhões de habitantes, manter sob controle ou erradicar doenças imunopreviníveis só se torna possível através da imunização (Brasil, 2003).

Sendo uma intervenção de saúde pública segura, econômica e efetiva, a vacinação além de prevenir, melhora a qualidade de vida, principalmente de populações com maior vulnerabilidade social. Sabe-se que crianças não vacinadas estão mais suscetíveis à morbimortalidade, desta maneira, a vacinação torna-se obrigatória em todo território nacional, segundo a Lei $\mathrm{n}^{\circ} 6.259$ regulamentada pelo Decreto $\mathrm{n}^{\mathrm{o}} 78.231$ 4, de agosto de 1976. Sendo a obrigatoriedade da vacinação de menores reforçada pelo Estatuto da Criança e do Adolescente (ECA) - Lei $n^{\circ} 8.069 / 90$ 5, que visa estabelecer o direito e proteção integral a esta faixa etária (Silva, et al., 2018; Barbieri \& Couto \& Aith, 2017).

O calendário de imunização vigente no Brasil, segundo o Ministério da Saúde (MS) preconiza que a criança até dois anos de idade seja submetida a pelo menos 22 procedimentos invasivos por conta do processo vacinal. Já na rede privada, onde o calendário é construído através das recomendações da Sociedade Brasileira de Imunização (SBIM) e Sociedade Brasileira de Pediatria (SPB), o número de vacinas é maior. A imunização da criança inicia-se na maternidade, onde a mesma terá seu primeiro contato com dor, desta maneira, torna-se importante e imprescindível a atenção na realização da primeira vacina ao bebê, isto porque caso seja traumático em um primeiro momento, a dor torna-se mais evidente no próximo ato vacinal (Erkul \& Efe, 2017).

A dor é definida como uma experiência subjetiva que pode estar ou não associada a um dano tecidual real ou potencial (Silva \& Ribeiro-Filho, 2011). A vacinação é a fonte de dor mais comum durante a infância, sendo fonte de sofrimento para crianças que são submetidas a este ato, e para pais que se culpabilizam pela execução da vacina (Fontes, et. al, 2013). Contudo, a dor tem sido negligenciada por profissionais de saúde, devendo os mesmos atentar-se mais para o manejo da dor, a fim de criar estratégias que visem sua minimização durante o processo de imunização. 
Desta maneira, os métodos não farmacológicos para alívio da dor de lactentes durante o momento da vacinação surgem para ampliar o leque de possibilidades de técnicas analgésicas, a qual o profissional deve apropriar-se em seu cotidiano. Isto pois, quando não dado a devida atenção para a dor do lactente, o mesmo pode ter consequências maléficas a curto, médio e longo prazo (García, et al., 2015).

Métodos como: a amamentação, ingesta de soluções açucaradas, manobras de distração, estimulação tátil e técnicas diferenciadas de administração da vacina, são medidas que auxiliam o profissional a atenuar a dor sentida pela criança no momento da aplicação de vacina (García, et al., 2015). Quanto a aplicabilidade da amamentação como analgesia, estudo aponta que é eficaz, uma vez que, a duração do choro significativamente menor, houve menor escore de dor, evitando do aumento da frequência cardíaca e da queda de oxigênio quando comparado a crianças que não utilizaram da técnica durante a aplicação de vacinas (Erkul \& Efe, 2017).

Outra estratégia não farmacológica para alívio da dor durante o processo vacinal, é o contato pele a pele, um dos pilares do Método Canguru, ainda que suas evidências sejam limitadas, quando se diz respeito a crianças com mais idade, sabe-se que o contato pele a pele promove a redução do nível de estresse e atua regulando o sistema tátil e proprioceptivo (Pandita, et al., 2018).

Devido ao fato do número de estudos sobre medidas para reduzir a dor do lactente durante a vacinação ser escasso, bem como, o sentimento das mães ao submeterem seus filhos a um procedimento doloroso, este estudo teve como objetivo compreender quais as percepções atribuídas pelos pais em relação ao manejo da dor durante a vacinação de seu filho, em uma clínica privada de vacinação do município de Florianópolis. Desta forma, a pergunta que fomentou esta pesquisa é: Qual a percepção das mães em relação ao uso de medidas não farmacológicas ao vacinar seus filhos?

\section{Metodologia}

Trata-se de uma pesquisa com abordagem qualitativa, o qual utilizou a Teoria Fundamentada nos Dados (TFD), que se baseia em um conjunto de procedimentos sistematizados para fim de desenvolver uma teoria acerca de um fenômeno, partindo da coleta de dados e sua análise simultaneamente (Strauss, 2002; Corbin, 2008).

O presente estudo atendeu aos preceitos éticos previstos na Resolução nº466/2012 do Conselho Nacional de Saúde. Sendo também aprovado pelo Comitê de Ética em Pesquisas com Seres Humanos da Universidade Federal de Santa Catarina, segundo o Parecer no 3.433.923 e CAAE: 09567419.9.0000.0121.

A coleta de dados foi realizada durante o período de junho a agosto de 2019, em uma clínica de imunização privada localizada no município de Florianópolis/SC. Desta forma, apresentou-se a proposta de pesquisa para os gestores da instituição, solicitando autorização para abordar individualmente e pessoalmente os pais e/ou cuidadores de crianças submetidas a vacinação.

Assim, a coleta dos dados foi realizada com mães de crianças que foram submetidas a vacinação, através de entrevistas semi-estruturadas, individuais e registradas por meio de gravação de áudio, sendo dividida em dois momentos, pré e pós aplicação. Tendo como pergunta inicial "Como você se sente no momento da aplicação da vacina na criança?". Levando em conta o sigilo recomendado, considerando o respeito pela dignidade humana e proteção dos participantes apareceram com a letra $\mathrm{M}$ representando as mães, seguindo numeradas conforme a ordem das entrevistas (M1, M2, M3...).

Os critérios para inclusão neste estudo foram pais e/ou cuidadores de crianças com idades entre 02 meses a 02 anos, submetidas as vacinações previstas no calendário recomendado pela Sociedade Brasileira de Imunização (SBIM). Foram excluídos deste estudo, pais e/ou cuidadores de crianças com mais de 02 anos de idade.

A amostra do estudo foi composta por um grupo amostral, composto por 10 mães de crianças com idades entre 02 meses até 02 anos de idade. A entrevista ocorreu dentro da sala de vacinação, garantindo privacidade do entrevistado, tendo 
como duração média de 04 minutos.

O método de análise seguiu conforme preconiza TFD, iniciando com a codificação aberta, codificação axial e por fim integração. Durante a codificação aberta os dados coletados na entrevista foram apreciados de forma cuidadosa, uma vez que, após realizado o desmembramento dos dados, começam a surgir códigos que são agrupados conforme similaridade ou diferença, conforme mostra a Tabela 1.

Tabela 1 - Codificação aberta.

\begin{tabular}{|l|l|}
\hline \multicolumn{1}{|c|}{ Dados } & \multicolumn{1}{|c|}{ Códigos } \\
\hline $\begin{array}{l}\text { Fala um pouquinho de como foi a sua percepção } \\
\text { hoje de ele ta no seu colo, tá no seio materno }\end{array}$ & $\begin{array}{l}6.1 \text { Percebeu que amamentando o bebê ficou mais calmo } \\
\text { e distraído. } \\
\text { Eu senti ele mais calmo, senti ele distraído também, } \\
\text { porque como eu to perto, o cheiro de leite né (risos), } \\
\text { isso distrai um tanto ele, e eu achei que foi mais } \\
\text { tranquilo, foi bem mais tranquilo. (M6) }\end{array}$ \\
\hline
\end{tabular}

Fonte: Autores.

A Tabela 1 retrata, na primeira coluna, um trecho da entrevista realizada com umas das participantes do estudo, na coluna seguinte, após avaliação minuciosa dos dados coletados no trecho em questão, tem-se os códigos gerados, conforme preza a etapa de codificação aberta da TDF.

Por seguinte, na etapa de codificação axial, estes dados foram reagrupados de maneira a formar grupos maiores, reunindo dados que se assemelham para dar origem a um conceito. Essas conexões entre categoria e subcategoria possibilitam uma melhor visão sobre o fenômeno emergido. Desta forma, as Tabelas 2 e 3 demonstram a partir de uma Entrevista M4, como realizou a etapa de codificação axial.

Tabela 2 - Elaboração de conceitos.

\begin{tabular}{|c|c|}
\hline Código preliminares & Conceito \\
\hline $\begin{array}{l}\text { 4.36 Acreditando que a com a amamentação é um } \\
\text { processo tranquilo para criança } \\
4.3 \text { Acreditando em situação de desconforto a criança } \\
\text { se acalma no peito } \\
4.15 \text { Sabendo que por ele estar com a mãe, no colo, ele } \\
\text { já está mais seguro }\end{array}$ & $\begin{array}{l}\text { Considerando o método da amamentação eficaz no } \\
\text { alívio da dor }\end{array}$ \\
\hline
\end{tabular}

Fonte: Autores. 
Tabela 3 - Elaboração de categorias através de subcategorias.

\begin{tabular}{|l|l|}
\hline \multicolumn{1}{|c|}{ Subcategorias } & \multicolumn{1}{c|}{ Categoria } \\
\hline $\begin{array}{l}\text { Tornando tranquilo o momento da vacinação; } \\
\text { Notando diferença na duração do choro da criança. }\end{array}$ & $\begin{array}{l}\text { Reconhecendo o método da amamentação como sendo } \\
\text { eficaz no alívio da dor }\end{array}$ \\
\hline
\end{tabular}

Fonte: Autores.

A Tabela 2 apresenta alguns dos códigos gerados na primeira etapa de codificação, que após nova análise são agrupados de acordo com suas semelhanças, gerando assim um conceito, que geram as categorias e subcategorias, conforme foi apresentado na Tabela 3.

Após a formação destes grupos, optou-se por trabalhar no modelo paradigmático, composto por três principais etapas, sendo elas: condição, ação-interação e consequência (Strauss, 2002; Corbin, 2008).

\section{Resultados}

Através da realização do processo de análise e integração dos dados emerge o seguinte fenômeno: "Percepção das mães ao utilizar a amamentação como método não farmacológico para alívio da dor em crianças submetidas à vacinação", fomentado por três categorias e cinco subcategorias conforme tabela a seguir (Tabela 4).

Tabela 4 - Categorias e subcategorias.

\begin{tabular}{|c|c|}
\hline CATEGORIA & SUBCATEGORIA \\
\hline $\begin{array}{l}\text { CONDIÇÃO } \\
\text { Preocupando-se com a dor do bebê ao submetê-lo a } \\
\text { procedimentos invasivos }\end{array}$ & $\begin{array}{l}\text { - Emergindo sentimentos ruins ao estar em uma } \\
\text { sala de vacinação; } \\
\text { - Vivenciando a dor junto com o bebê. }\end{array}$ \\
\hline $\begin{array}{l}\text { AÇÃO-INTERAÇÃO } \\
\text { Reconhecendo o método da amamentação como sendo } \\
\text { eficaz no alívio da dor }\end{array}$ & $\begin{array}{l}\text { - Tornando tranquilo o momento da vacinação; } \\
\text { - Notando diferença na duração do choro da } \\
\text { criança. }\end{array}$ \\
\hline $\begin{array}{l}\text { CONSEQUÊNCIA } \\
\text { Dando continuidade à aplicação do método não } \\
\text { farmacológico durante a vacinação em outros } \\
\text { contextos. }\end{array}$ & $\begin{array}{l}\text { - Utilizando a amamentação durante a vacinação } \\
\text { em outros locais. }\end{array}$ \\
\hline
\end{tabular}

Fonte: Autores.

A tabela 4 apresenta as três categorias resultantes do estudo, onde cada uma pertence a uma das principais etapas do método escolhido, assim como suas cinco subcategorias, resultantes do agrupamento dos códigos obtidos nas entrevistas.

A amostra do estudo foi composta por um grupo amostral constituído de 10 mães, sendo a média de idade 37 anos, destas apenas duas eram multíparas. Da totalidade do grupo amostral, somente uma havia realizado o desmame parcial do lactente.

A categoria condição intitulada como "Preocupando-se com a dor do bebê ao submetê-lo a procedimentos invasivos", é composta por 03 subcategorias, das quais apresentam os sentimentos e anseios das mães em estar presentes na sala de vacinação e submeter seus filhos a um procedimento doloroso. 
Na primeira subcategoria, "Emergindo sentimentos ruins ao estar em uma sala de vacinação", as mães demonstram sentimentos como medo e pânico por estarem dentro de uma sala de vacinação tendo que participar de um momento que causará dor aos seu filho.

"As primeiras um pânico, depois vai tranquilizando, é para o bem dele, não tem o que fazer, procuro me acalmar o máximo para acalmar ele" (M6).

"Eu fico assustada, fico com bastante medo, mas é o melhor para ele, então faz parte" (M3).

"Meu filho mais velho, quando a gente vacinava não deixavam ficar no peito, então ficava incomodada, não gostava de estar junto, ficava no lado de fora, porque tinha que segurar a criança, a forma que eu não concordo em vacinar entendeu?" (M4).

A segunda subcategoria, "Vivenciando a dor junto com o bebê", as mães falam sobre sentir pena, dó principalmente e se culpabilizam pois estão sujeitando seus filhos a um procedimento invasivo que culminará em dor.

“Peninha dela né. A gente já sente tanta dor, ainda eles tão pequenininhos né...” (M1).

“[...] ah dá dózinha né...Assim tranquila de estar fazendo o bem, mas da pena por causa da dor” (M2).

“[...] eu sinto que dá uma dó, é como se eu sentisse dorzinha com ele no choro dele e ele sempre chora” (M7).

O segundo componente ação-interação, composto por duas subcategorias, foi denominado de "Reconhecendo o método da amamentação como sendo eficaz no alívio da dor", trata da percepção das mães logo após a realização da vacinação com uso da amamentação como método analgésico. Nesta categoria, o fato de estar em uma sala de vacinas e submeter a criança a uma vacinação passa a ser algo tranquilo, o choro do bebê que antes era algo que lhes traziam sentimentos ruins passa a ser mais ameno.

A primeira subcategoria intitulada de "Tornando tranquilo o momento da vacinação" diz respeito a percepção das mães ao estarem participando de forma ativa no momento do procedimento invasivo. O fato de estarem amamentando e em contato pele a pele, traz segurança ao binômio.

"Percebi que ao amamentar me senti mais confortável [...] É bem melhor, senti mais segurança ficando com ela no meu colo" (M1).

"Foi uma experiência melhor, do que ficar com ele na cama. Eu me senti mais segura (M7)"

"Foi melhor também, senti que posso dar mais segurança pra ela, e ver ela chorando menos foi bem melhor, do que ver ela na maca e berrando, muito ruim (M8)"

A segunda subcategoria, "Notando diferença na duração do choro da criança", traz a percepção das mães quanto ao comportamento dos filhos durante o procedimento invasivo onde foi utilizado como analgesia a amamentação.

"Amamentando ele chorou bem menos, bem mais tranquilo. Na primeira vez que eu estava amamentando ele nem 
chorou" (M3).

"É, foi mais tranquilo, parece que chorou menos mesmo [...] ficou bem tranquilo, foi bem bom" (M7). "Ela parece que fica mais confortável assim no peito, chora um pouco menos. menos traumático" (M8).

Por fim, a última categoria nomeada como "Dando continuidade à aplicação do método não farmacológico durante a vacinação em outros contextos" diz respeito a satisfação e o desejo das mães de dar continuidade na realização da amamentação como um método de alívio da dor do lactente durante um procedimento invasivo. Esta categoria é contemplada com uma subcategoria "Utilizando a amamentação durante a vacinação em outros locais" que se refere ao desejo das mães em dar continuidade à amamentação em outros locais, não se restringindo apenas a rede de clínicas privadas.

"Pretendo continuar a fazer as vacinas amamentando, inclusive vou tentar até no posto de saúde" (M1).

"Na outra clínica não me ofereceram essa prática, só aqui que me ofereceram. Gostei, na próxima vez vou tentar de novo, vamos ver, mas eu gostei, achei bem interessante" (M9).

"Sim, não só pretendo continuar, como falarei para minhas colegas que tiveram filhos recentes utilizarem também" (M10).

O estudo não apresentou nenhuma desistência por parte dos entrevistados, desta maneira a partir da análise dos resultados presentes pode-se concluir que a amamentação durante a imunização da criança, trouxe novo significado para as mães de como é estar em uma sala de vacinas e participar do momento da vacinação. Além disso, é notável que as mães percebem um comportamento diferenciado do bebê ao realizar o procedimento invasivo com auxílio da amamentação. Ainda assim, pode-se perceber que infelizmente ainda não são todos os locais que ofertam métodos não farmacológicos para alívio da dor de lactentes submetidos à vacinação.

\section{Discussão}

Este estudo traz como resultado aspectos vivenciados por mães e bebês no processo de imunização. Presenciar o sofrimento e/ou a dor de um filho, realmente não é uma tarefa fácil para as mães. Desde o nascimento a presença materna é fortemente marcada, de tal modo que a criança se torna incapaz de se desenvolver satisfatoriamente sem a criação do vínculo mãe-bebê. Este vínculo torna-se tão forte de maneira com que as mães passam a se colocar no lugar de seus filhos, a fim de atender às necessidades por eles apresentadas (Mozzaquatro \& Arpini \& Polli, 2016).

São escassas as evidências na literatura que abordam a dor das mães ao assistirem seus filhos em um procedimento e os sentimentos das mães ao adentrarem em uma sala de vacinação. Os estudos relacionados à dor em recém-nascidos estão relacionados a períodos de internação hospitalar, principalmente em Unidades Neonatais. Desta forma, descrevem que a Unidade de Terapia Intensiva Neonatal (UTIN) é vista pelas mães como um ambiente ameaçador, isto porque, além de acontecer a separação do binômio, o bebê será submetido a procedimentos dolorosos. Percebemos semelhanças em relação a sala de vacinação, onde ainda na grande maioria das vezes, a criança é separada de sua mãe sendo contida em uma maca e submetida a um procedimento invasivo que culminará em dor (Rodrigues \& Moreira, 2012).

Esses estudos fundamentam as falas expostas na categoria condição, onde as mães preocupam-se com a dor do bebê e as vivem junto com ele. Todos os sentimentos demonstrados nessa categoria são ressignificados na categoria ação-interação 
após a implementação da amamentação dentro do ambiente vacinal. Isto porque, o aleitamento materno além de ser uma das etapas mais importantes do processo reprodutivo da mulher, provê benefícios tanto para a criança quanto para mãe (Martins \& Santana, 2013).

A amamentação promove a liberação de ocitocina na corrente sanguínea, isto acontece porque a sucção estimula a hipófise liberar este hormônio (Antunes, et al., 2008). Além de auxiliar como uma facilitadora do processo de contração uterina e ejeção do leite durante a lactação, a ocitocina auxilia regulando também o comportamento maternal. As altas taxas de ocitocina e a diminuição da ativação da amígdala, são capazes de reduzir o medo, o estresse, a ansiedade e mau humor (Oliveira, 2014).

Corroborando com os resultados das subcategorias, as mães que antes referiam medo e desconforto, relatam que o momento da vacinação se tornou tranquilo após a utilização da amamentação. Desta maneira, deve-se ressaltar que os benefícios do leite materno também se estendem a criança, que ao buscar o seio materno para além de alimentar-se, o bebê encontra segurança e consolação. Dentre os métodos não farmacológicos para alívio da dor de lactentes submetidos à vacinação, amamentar acaba por ser mais eficaz quando comparado aos demais métodos, tais como administração de leite humano e soluções doces, uso de chupetas, dentre outros (García, et al., 2015).

Esta eficiência se dá através de um mecanismo múltiplo que envolve, conforto físico, sucção, tração, ingesta de sabor doce e outras substâncias que de maneira conjunta minimizam a dor ocasionada pelo procedimento invasivo (Erkul \& Efe, 2017). De acordo com García, et al., (2015) para o sucesso da analgesia através da amamentação, recomenda-se que a criança pegue o seio de forma efetiva antes da realização da vacinação, e que este ato prossiga até depois do término do procedimento.

Em recém-nascidos internados, a amamentação durante procedimentos invasivos, é capaz de promover um menor escore de dor, impedimento do aumento da frequência cardíaca e da queda de oxigênio e redução do choro (Erkul \& Efe, 2017; Chittaluri \& Rani, 2017). Para RN internados, é comprovado que a amamentação está intimamente ligada com o Método Canguru, uma vez que, amamentar promove o contato pele a pele. Este contato auxilia reduzindo o nível de estresse e regulando o sistema tátil e proprioceptivo durante a realização da vacina (Pandita, et al., 2018).

Sendo assim, estes estudos vão de encontro à percepção das mães desta pesquisa, uma vez que, as mesmas notaram diferença na duração do choro da criança quando utilizado os métodos não farmacológicos para alívio da dor. Através desta constatação, na categoria consequência, as mães expressam a satisfação e a vontade de dar continuidade a amamentação durante procedimentos invasivos.

Percebemos que a adoção desta prática em uma clínica particular tem estimulado a busca pela satisfação dos clientes, onde a experiência emocional/sentimental é um fator de suma importância na satisfação do consumidor. Desta forma, os sentimentos positivos que surgem durante e após a experiência do consumidor contribuem e influenciam na satisfação do cliente (Westbrook \& Oliver, 1991).

Deve-se valorizar a importância dos profissionais supervisores do serviço e da sala de vacinação, sendo eles, enfermeiros e farmacêuticos que através de seus conhecimentos práticos-teóricos, acabam sendo os profissionais de referência para incentivar mudanças de rotinas dentro deste ambiente.

\section{Conclusão}

Perceber a dor do lactente é uma função essencial na prática da equipe de enfermagem. Olhar o ser humano de forma holística é compreender também a família daquele que está recebendo os cuidados. O que seria uma simples aplicação de vacina passa a ser uma consulta de enfermagem, onde deveriam ser levadas em conta angústia da criança ao ser submetida à um procedimento doloroso, a percepção da mãe quanto a isto e a orientação de métodos que visem tornar a vacinação um procedimento não mais doloroso, mas sim, procedimento agradável para todos os envolvidos. 
As mães que antes atuavam apenas em segundo plano, agora passam a ser um dos atores principais da vacinação, sendo imprescindível sua presença e colaboração durante o ato vacinal. Pode-se contemplar a amamentação não só como uma forma de alimentação para o lactente, mas sim como método analgésico que auxilia na redução da dor ocasionada pela vacinação.

Apesar de ser um tema ainda pouco explorado, a utilização de métodos não farmacológicos para alívio da dor em lactentes submetidos à vacinação, bem como, a percepção que os pais e/ou cuidadores têm sobre este processo, possuem um valor ímpar para a pesquisa em enfermagem, uma vez que, os assuntos aqui tratados são utilizados no aperfeiçoamento profissional.

\section{Referências}

Antunes, L. dos S., Antunes, L. A. A., Corvino, M. P. F. \& Maia, L. C. (2008). Amamentação natural como fonte de prevenção em saúde. Ciência \& Saúde Coletiva, 13(1), 103-109. https://doi.org/10.1590/S1413-81232008000100015

Barbieri, C. L. A., Couto, M. T. \& Aith, F. M. A. (2017). A (não) vacinação infantil entre a cultura e a lei: os significados atribuídos por casais de camadas médias de São Paulo, Brasil. Cadernos de Saúde Pública, 33(2), 1-11. https://doi.org/10.1590/0102-311X00173315

Brasil. (2003). Programa Nacional de Imunizações: 30 anos. Brasília: Ministério da Saúde. Secretaria de Vigilância em Saúde.

Chittaluri, V., \& Rani, S. R. (2017). Effectiveness of Breast Feeding on Pain Perception During Vaccination among Infants. International Journal of Nursing Education, 9(2), 52-56. https://doi.org/10.5958/0974-9357.2017.00035.6

Erkul, M., \& Efe, E. (2017). Efficacy of Breastfeeding on Babies' Pain During Vaccinations. Breastfeeding Medicine, 12(2), 110-115. https://doi.org/10.1089/bfm.2016.0141

Fontes, V. S., Ribeiro, C. J. N., Dantas, R. A. N. \& Ribeiro, M. do C. de O. (2018). Pain relief strategies during immunization. [Eletronic version] Brazilian Journal Of Pain, 1(3), 1-4. doi: 10.5935/2595-0118.20180051

García Sánchez, N., Merino Moína, M., García Vera, C., Lacarta García, I., Carbonell Muñoz, L., Pina Marqués, B., Álvarez García, F.J., \& Arístegui Fernández, J. (2015). Alivio del dolor y el estrés al vacunar. Síntesis de la evidencia: recomendaciones del Comité Asesor de Vacunas de la AEP. Pediatría Atención Primaria, 17(68), 317-327. https://dx.doi.org/10.4321/S1139-76322015000500006

Martins, M. Z. (2013). Benefícios da amamentação para saúde materna. Interfaces Científicas - Saúde E Ambiente, 1(3), 87-97. https://doi.org/10.17564/2316$3798.2013 \mathrm{v} 1 \mathrm{n} 3 \mathrm{p} 87-97$

Mozzaquatro, C. de O., Arpini, D. M. \& Polli, R. G. (2016). Relação mãe-bebê e promoção de saúde no desenvolvimento infantil. Psicologia em Revista, 21(2), 333-346. https://doi.org/10.5752/P.1678-9523.2015V21N2P333

Oliveira, M. P. de. (2014). A Ocitocina E Suas Inúmeras Aplicações. Monografia (Especialização). Curso de Farmacia, Farmácia Clínica e Atenção Farmacêutica, Universidade Católica de Goiás \& Instituto Pharmacológica, Goiás, Brasil.

Pandita, A., Panghal, A., Gupta, G., Verma, A., Pillai, A., Singh, A., \& Naranje, K. (2018). Is kangaroo mother care effective in alleviating vaccination associated pain in early infantile period? A RCT. Early Human Development, 127, 69-73. https://doi.org/10.1016/j.earlhumdev.2018.10.001

Rodrigues, L. M. \& Moreira, P. L. (2012). Tornar-se pai vivenciando a internação do filho em Unidade de Terapia Intensiva Neonatal. J Health Sci Inst., 30(2), 227-230.: http://repositorio.unip.br/wp-content/uploads/2020/12/V30_n3_2012_p227a230.pdf

Silva, F. de S., Barbosa, Y. C., Batalha, M. A., Ribeiro, M. R. C., Simões, V. M. F., Branco, M. dos R. F. C., Thomaz, E. B. A. F., Queiroz, R. C. de F., Araújo, W. R. M. \& Silva, A. A. M. da. (2018). Incompletude vacinal infantil de vacinas novas e antigas e fatores associados: coorte de nascimento BRISA, São Luís, Maranhão, Nordeste do Brasil. Cadernos de Saúde Pública, 34(3), 1-21. https://doi.org/10.1590/0102-311X00041717

da Silva, J. A., \& Ribeiro-Filho, N. P. (2011). A dor como um problema psicofísico. Revista Dor, 12(2), 138-151. doi: 10.1590/S180600132011000200011

Strauss, A. \& Corbin, J. (2002) Bases de la Investigación Cualitativa. Técnicas y procedimientos para desarrollar la Teoría Fundamentada. Colômbia: Editorial Universidad de Antioquia, publicado por acordo com Sage Publications, Inc. Universidad de Antioquia.

Strauss, A., \& Corbin, J. (2008). Pesquisa Qualitativa: técnicas e procedimentos para o desenvolvimento de teoria fundamentada. Artmed.

Winnicott, D. W. (2000). A preocupação materna primária. In: D. W. Winnicott (Ed.) Da pediatria à psicanálise: Obras escolhidas. Editora Imago.

Westbrook, R. A. \& Oliver, R. L. (1991). The dimensionality of consumption emotion patterns and consumer satisfaction. Journal of Consumer Researchn, 18, 84-91. https://doi.org/10.1086/209243 